Egyptian

Orthodontic Journal

\title{
EFFECT OF DIFFERENT BONDING TECHNIQUES ON THE DEBONDING CHARACTERISTICS OF CERAMIC BRACKETS
}

\author{
Moustafa A. Tageldin ${ }^{1}$, Hanan A. Ismail' \\ Mohamed I. Mowafy ${ }^{3}$, \\ ABSTRACT: \\ Objective: To evaluate and compare shear bond strength and \\ debonding characteristics of ceramic brackets using different \\ bonding techniques. Materials and Methods: Ninety six premolar \\ teeth were randomly divided into six groups: 1) Transbond $X \mathcal{T}$ \\ with 5 seconds etch, 2) Transbond XT with 15 seconds etch, \\ 3) Transbond XT with Transbond Plus Self - Etching Primer, \\ 4) Vertise Flow with 5 seconds etch, 5) Vertise Flow with \\ 15 seconds etch, 6) Vertise Flow with Transbond Plus \\ Self-Etching Primer. In each group 16 bracketed teeth with \\ Inspire Ice bracket, 8 of which were debonded by universal testing \\ machine to determine the shear bond strength. The other 8 ceramic \\ brackets were removed with plastic debonding plier. All teeth were \\ examined under a stereomicroscope, the adhesive remnant index \\ and bracket fracture were assessed. Results: The mean SBS \\ for group I to VI was $(1.78 \pm 0.78,14.5 \pm 5,12.52 \pm 3.69$, \\ $4.15 \pm 2.35,13.26 \pm 4.46,6.78 \pm 3.51$,) MPa respectively. Bracket \\ fractures were noted in 10 out of 48 samples after debonding with \\ plier. Groups II and $V$ exhibited the higest SBS and the highest \\ ratio of bracket fracture. Conclusion: Using 15 seconds etching \\ with either Transbond XT or Vertise Flow gave acceptable SBS.
}

1- Instructor, Orthodontics department, Faculty of Dentistry, Alexandria University

2- Professor of Orthodontics Faculty of Dentistry, Alexandria University

3- Assistant professor of Orthodontics, Faculty of Dentistry, Alexandria University 
Egyptian

Orthodontic Journal

Self-etching primer gave lower SBS but within the acceptable clinical range. Decreasing etching time to 5 seconds didn't give clinically acceptable SBS.

\section{INTRODUCTION}

The ceramic brackets were introduced in the 1980s. ${ }^{1}$ They are more esthetic than metal brackets, and unlike plastic brackets, they resist staining and discoloration. ${ }^{2}$ The Inspire Ice bracket (Ormco, Orange, Calif.) is a monocrystalline ceramic bracket that features mechanical retention. Recent literature reports that even mechanically retained ceramic brackets have greater bond strengths than metal brackets. ${ }^{3-5}$

Enamel damage and bracket fracture are common complications with debonding of ceramic brackets, which are attributed to the high bond strength combined with the low fracture toughness of ceramics. ${ }^{3,4,6}$ Bracket breakage may result in eye injury, ingestion, or aspiration of bracket fragments. In addition, the remaining bracket sometimes demands removal with a high speed handpiece diamond bur which may sometimes damage the enamel. ${ }^{7}$

The force applied during debonding ceramic brackets is influenced by the bracket retention mechanism, the composition of the adhesive, the method of enamel conditioning and the method of debonding. ${ }^{8}$ The lower the force used during debonding, the less is the likelihood for bracket failure. ${ }^{9}$

A self-etching primer (SEP) combines the etching, rinsing, and priming steps into a single step resulting in a less technique sensitive bonding procedure. ${ }^{10}$ This lead to the enhancement of both time and cost-effectiveness to the clinician and, indirectly, to the patient. ${ }^{11}$ Transbond Plus SEP, was developed especially for orthodontic bonding. However, whether SEPs or conventional etchants are better, and the best SEP, acid, concentration and etching time, remain to be undetermined. ${ }^{12}$

Lately, a new self-adhering flowable resin composite, Vertise Flow (Kerr, Orange, CA, USA), has been launched into the dental market. Vertise Flow has been proposed as a self-adhering restorative material. ${ }^{13}$ The simplified handling of Vertise Flow makes this material attractive for bonding orthodontic brackets. 
Egyptian

Orthodontic Journal

The aim of the present in vitro study was to test different enamel conditioning methods and different adhesives to obtain optimum shear bond strength and the best debonding characteristics regarding bracket fracture and enamel damage, reducing the most common problems encountered during ceramic brackets debonding.

\section{MATERIALS AND METHODS}

Ninety six extracted premolars for orthodontic purposes were divided randomly into 6 groups each consists of 16 teeth and were kept in sealed boxes containing saline solution. The Inspire Ice brackets were bonded to the teeth as following:

Group I: Enamel surface was etched for 5 seconds using 37\% phosphoric acid gel, rinsed with water for 15 seconds and air-dried for 15 seconds. Transbond XT primer was applied to the enamel surfaces using disposable brush.

Group II: Same as group I but with 15 seconds etching

Group III: Transbond plus SEP was rubbed on the enamel surface for 5 seconds

For groups I, II and III: Inspire Ice brackets were bonded to the enamel surface using Transbond XT light cure orthodontic adhesive. The adhesive was applied to the bracket, then the bracket was put on the middle third of the crown using bracket holder and pressed firmly on the tooth surface in order that the excessive adhesive to be pushed out and then removed by dental probe. The adhesive was then cured using light emitting diode curing light for 20 seconds.

Group IV: Enamel surface was etched for 5 seconds using 37\% phosphoric acid gel, rinsed with water for 15 seconds, and air-dried for 15 seconds.

Group V : Same as group IV but with 15 seconds etching.

Group VI: Transbond plus SEP was rubbed on the enamel surface for 5 seconds.

For groups IV, V and VI: Inspire Ice brackets were bonded to the enamel surface using Vertise flow light cure flowable composite. A small 
amount of resin composite was placed onto the bracket base, then the bracket was put on then middle third of the crown using bracket holder and pressed firmly on the tooth surface in order that the excessive adhesive pushed out and then removed by dental probe. The adhesive was then cured using light emitting diode curing light for 20 seconds.

All the samples were thermocycled in water bath for 1000 cycles alternating between $5{ }^{\circ} \mathrm{C}$ and $55^{\circ} \mathrm{C}$ dwell time 30 seconds. All teeth were mounted vertically using a surveyor in self-cure acrylic resin blocks covering the root of the teeth to the cemento-enamel junction.

Half of the sample of each group (8 teeth) was subjected to a shear load using universal testing machine with crosshead speed adjusted to 0.5 $\mathrm{mm} / \mathrm{min}$ to determine the shear bond strength (SBS) (figure 1). The other half of the sample ( 8 teeth) was debonded by hand using the plastic plier recommended by the manufacturer.

All the debonded samples were examined under a stereomicroscope to assess the adhesive remnant index. For the brackets debonded by the plier, the number of bracket fractured was recorded.

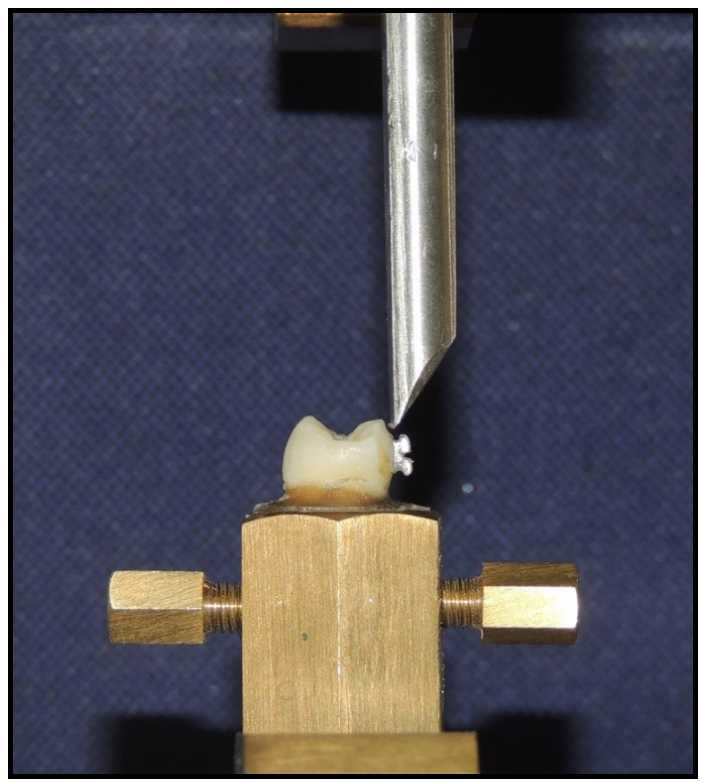

Figure 1: View of the blade of the universal testing machine paralleled to the surface of the tooth at the bracket tooth interface. 
Egyptian

Orthodontic Journal

\section{RESULTS}

\section{I- Shear bond strength:}

The mean SBS for the six groups is shown in (Table 1). The results of Mann Whitney test showed that the SBS for the group I is significantly lower than the group II and III, while there was no significant difference between group II and III. The SBS of group V was significantly higher than than group IV and VI, while there was no significant difference between group IV and VI. When comparing the SBS between group II and group V, No statistically significant difference was found.

Table 1: Comparison of shear bond strength in MPa between different study groups

\begin{tabular}{|c|c|c|c|c|c|c|}
\hline & \multicolumn{3}{|c|}{ Transbond XT } & \multicolumn{3}{c|}{ Vertise Flow } \\
\hline & Group I & Group II & Group III & Group IV & Group V & Group VI \\
\hline Mean \pm SD & $1.78 \pm 0.78$ & $14.50 \pm 5.0$ & $12.52 \pm 3.69$ & $4.15 \pm 2.35$ & $13.26 \pm 4.46$ & $6.78 \pm 3.51$ \\
\hline $\mathbf{p}_{1}$ & & $0.001^{*}$ & $0.001^{*}$ & & $0.001^{*}$ & 0.074 \\
\hline $\mathbf{p}$ & \multicolumn{5}{c|}{$\mathrm{p}_{2}=0.401, \mathrm{p}_{3}=0.012^{*}, \mathrm{p}_{4}=0.636$} \\
\hline
\end{tabular}

$\mathrm{p}_{1}$ : $\mathrm{p}$ value for Mann Whitney test for comparing between group1 with group 2 and 3, also for comparing between group 4 with 5 and 6

$\mathrm{p}_{2}: \mathrm{p}$ value for Mann Whitney test for comparing between group2 and 3

$\mathrm{p}_{3}$ : $\mathrm{p}$ value for Mann Whitney test for comparing between group 5 and 6

$\mathrm{p}_{4}$ : $\mathrm{p}$ value for Mann Whitney test for comparing between group 2 and 5

*: Statistically significant at $\mathrm{p} \leq 0.05$

\section{II- Adhesive remnant index:}

For the brackets debonded by the universal testing machine (Table 2), the Mann Whitney comparison of the ARI scores showed significant difference between 5 seconds etch, 15 seconds etch and SEP used with Transbond XT (group I, II and III respectively), while there was no significant difference between 5 seconds etch, 15 seconds etch and SEP used with Vertise Flow (group IV, V, VI respectively). Also, there was no significant difference between the 15 seconds etching time used with Transbond XT and Vertise Flow (group II and V). 


\section{Egyptian \\ Orthodontic Journal}

Table 2: Comparison of Adhesive Remnant Index among the six groups after debonding by the universal testing machine

\begin{tabular}{|c|c|c|c|c|c|c|c|c|c|c|c|c|}
\hline \multirow{3}{*}{ ARI } & \multicolumn{6}{|c|}{ Transbond XT } & \multicolumn{6}{|c|}{ Vertise Flow } \\
\hline & \multicolumn{2}{|c|}{ Group I } & \multicolumn{2}{|c|}{ Group II } & \multicolumn{2}{|c|}{ Group III } & \multicolumn{2}{|c|}{ Group IV } & \multicolumn{2}{|c|}{ Group V } & \multicolumn{2}{|c|}{ Group VI } \\
\hline & No. & $\%$ & No. & $\%$ & No. & $\%$ & No. & $\%$ & No. & $\%$ & No. & $\%$ \\
\hline $\mathbf{0}$ & 5 & 62.5 & 0 & 0.0 & 0 & 0.0 & 0 & 0.0 & 0 & 0.0 & 0 & 0.0 \\
\hline 1 & 3 & 37.5 & 1 & 12.5 & 5 & 62.5 & 4 & 50.0 & 4 & 50.0 & 5 & 62.5 \\
\hline 2 & 0 & 0.0 & 6 & 75.0 & 3 & 37.5 & 4 & 50.0 & 3 & 37.5 & 3 & 37.5 \\
\hline 3 & 0 & 0.0 & 1 & 12.5 & 0 & 0.0 & 0 & 0.0 & 1 & 12.5 & 0 & 0.0 \\
\hline Mean \pm SD & \multicolumn{2}{|c|}{$0.38 \pm 0.52$} & \multicolumn{2}{|c|}{$2.0 \pm 0.53$} & \multicolumn{2}{|c|}{$1.38 \pm 0.52$} & \multicolumn{2}{|c|}{$1.50 \pm 0.53$} & \multicolumn{2}{|c|}{$1.63 \pm 0.74$} & \multicolumn{2}{|c|}{$1.38 \pm 0.52$} \\
\hline $\mathbf{p}_{1}$ & & & \multicolumn{2}{|c|}{$0.001 *$} & \multicolumn{2}{|c|}{$0.005^{*}$} & & & \multicolumn{2}{|c|}{0.814} & \multicolumn{2}{|c|}{0.626} \\
\hline $\mathbf{p}$ & \multicolumn{12}{|c|}{$\mathrm{p}_{2}=0.036^{*}, \mathrm{p}_{3}=0.511, \mathrm{p}_{4}=0.215$} \\
\hline
\end{tabular}

$\mathrm{p}_{1}$ : $\mathrm{p}$ value for Mann Whitney test for comparing between group1 with group 2 and 3 , also for comparing between group 4 with 5 and 6

$\mathrm{p}_{2}$ : $\mathrm{p}$ value for Mann Whitney test for comparing between group2 and 3 $\mathrm{p}_{3}$ : $\mathrm{p}$ value for Mann Whitney test for comparing between group 5 and 6 $\mathrm{p}_{4}$ : $\mathrm{p}$ value for Mann Whitney test for comparing between group 2 and 5

*: Statistically significant at $\mathrm{p} \leq 0.05$

\section{III- Bracket fracture:}

The number of fractured brackets was recorded (Table 3). The highest percentage of bracket fracture was found in group II and V (Figure 2). The results of the Fisher exact test indicated no significant differences between all the groups regarding bracket fracture.

Table 3: Comparison between the different studied groups according to bracket fracture

\begin{tabular}{|c|c|c|c|c|c|c|c|c|c|c|c|c|}
\hline & \multicolumn{6}{|c|}{ Transbond XT } & \multicolumn{6}{|c|}{ Vertise Flow } \\
\hline & \multicolumn{2}{|c|}{ Group I } & \multicolumn{2}{|c|}{ Group II } & \multicolumn{2}{|c|}{ Group III } & \multicolumn{2}{|c|}{ Group IV } & \multicolumn{2}{|c|}{ Group V } & \multicolumn{2}{|c|}{ Group VI } \\
\hline & No. & $\%$ & No. & $\%$ & No. & $\%$ & No. & $\%$ & No. & $\%$ & No. & $\%$ \\
\hline Fracture & 0 & 0.0 & 3 & 37.5 & 1 & 12.5 & 1 & 12.5 & 3 & 37.5 & 2 & 25.0 \\
\hline No Fracture & 8 & 100.0 & 5 & 62.5 & 7 & 87.5 & 7 & 87.5 & 5 & 62.5 & 6 & 75.0 \\
\hline FEp1 & & & \multicolumn{2}{|c|}{0.200} & \multicolumn{2}{|c|}{1.000} & & & \multicolumn{2}{|c|}{0.569} & \multicolumn{2}{|c|}{1.000} \\
\hline FEp & \multicolumn{12}{|c|}{$\mathrm{p} 2=0.569, \mathrm{p} 3=1.000, \mathrm{p} 4=1.000$} \\
\hline
\end{tabular}

p1: $\mathrm{p}$ value for Fisher Exact test for comparing between group1 with group 2 and 3, also for comparing between group 4 with 5 and 6

p2: $p$ value for Fisher Exact test for comparing between group2 and 3

p3: p value for Fisher Exact test for comparing between group 5 and 6

p4: $p$ value for Fisher Exact test for comparing between group 2 and 5 
Egyptian

Orthodontic Journal

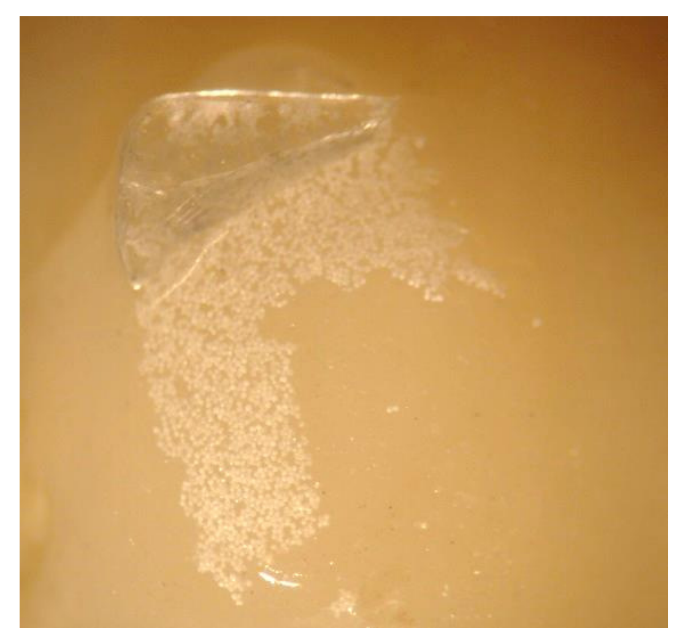

Figure 2: Stereomicroscope image showing enamel surface fragment of ceramic bracket.

\section{DISCUSSION}

Minimum bond strength for clinically acceptable bracket bonding was reported by Reynolds and von Fraunhofer to be $6-8 \mathrm{MPa}^{14,15}$ The maximum bond strength of an orthodontic bracket should be less than the breaking strength of enamel which is about $14 \mathrm{MPa} .{ }^{16}$ The finding of this study showed that 5 seconds etching is not enough to produce clinically acceptable SBS with both types of adhesives, Transbond XT and Vertise Flow.

When using Transbond XT adhesive, the SBS with SEP $(12.52 \pm 3.69) \mathrm{MPa}$ is lower than that with 15 seconds etching $(14.50 \pm 5.0) \mathrm{MPa}$ but with no statistically significant difference $(p=0.401)$. This result is consistent with the results of study carried out by Mirzakouchaki et al. ${ }^{17}$

Isman et $\mathrm{al}^{18}$ reported that Transbond XT with 15 seconds etching exhibited SBS $(9.86 \pm 3.20) \mathrm{MPa}$, and there were no statistically significant differences with Vertise Flow and 15 seconds etching $(7.89 \pm 1.17) \mathrm{MPa}$. These mean results are lower than that found in the present study; this may be due to the different types of brackets. But in this study also, there were no statistically significant differences when 15 seconds etching was used with both types of adhesives. 
Egyptian

Orthodontic Journal

Goracci et $\mathrm{al}^{19}$ reported mean SBS of $(11.70,8.98,2.99,6.56) \mathrm{MPa}$ for Transbond XT with 15 seconds etching, SEP, Vertise Flow without etching and for Vertise Flow with 15 seconds etching respectively, when bonding stainless steel brackets. The SBS for the Vertise Flow with 15 seconds etching is significantly lower than that of Transbond XT which is different from the present study. It has been stated that Vertise Flow can effectively bond to porcelain without the need for hydrofluoric acid etching and silane application ${ }^{13}$. This might affect the SBS of Ceramic brackets bonded with the new self-adhering flowable composite.

Microscopic observations of the failure sites added some useful information about the bond established by the tested materials. Failure at the bracket adhesive interface can indicate safe debonding and reduce the probability of enamel damage. ${ }^{20}$ However, the cleaning procedures to remove the residual adhesives are usually accompanied by a degree of enamel loss. ${ }^{21}$ The clean-up procedure of the adhesive after debonding may remove up to $55.6 \mu \mathrm{m}$ of surface enamel. ${ }^{22}$

In the current study, 5 seconds etching with Transbond XT, exhibited ARI scores 0 and 1 . This is expected, as mechanical retention created in the enamel was not enough. However, the ARI scores for the 5 second etching with Vertise Flow group ranged from 0 to 2 .

ARI Score 3, indicating all the adhesive remained on the tooth surface was only seen with groups II and V (15 seconds etching). That may be explained by their higher SBS. When using 15 seconds etching, there were no significant differences in ARI scores between Transbond $\mathrm{XT}$ and Vertise flow. Both groups exhibited the highest mean SBS and the highest ARI scores average.

The incidences of cohesive ceramic fractures were $37.5 \%$ for the 15 seconds etch with both Transbond XT and Vertise Flow, which is the highest among all the groups. This can be explained by the higher SBS exhibited by both groups. While group I showed no bracket fracture, may be due to the lower SBS needed to debond them. It is well documented that as the debonding forces increase, so does the frequency of enamel cracks and bracket fracture. ${ }^{23-25}$ 
SEP with Transbond XT was the group with an acceptable SBS and the least amount of bracket fracture. With only $12.5 \%$ incidence of bracket fracture, while 5 seconds etching with Vertise Flow showed the same amount of bracket fracture but the SBS is less than the optimum.

\section{CONCLUSION}

1. Using 15 seconds etching with either Transbond XT or Vertise Flow gave acceptable SBS.

2. Self-etching primer give lower SBS but within the acceptable clinical range.

3. Decreasing etching time to 5 seconds didn't give clinically acceptable SBS.

4. 15 seconds etching showed higher incidence of ARI scores 2 and 3, indicating greater amount of adhesive remained on the teeth.

5. The incidence of bracket fracture increases with higher SBS.

\section{REFERENCES}

1. Winchester LJ. Bond strengths of five different ceramic brackets: an in vitro study. Eur J Orthod. 1991;13(4):293-305

2. Kusy RP. Orthodontic biomaterials: from the past to the present. Angle Orthod. 2002;72(6):501-12

3. Joseph VP, Rossouw E. The shear bond strengths of stainless steel and ceramic brackets used with chemically and light-activated composite resins. Am J Orthod Dentofacial Orthop. 1990;97(2):121-5

4. Reddy YG, Sharma R, Singh A, Agrawal V, Agrawal V, Chaturvedi S. The Shear Bond Strengths of Metal and Ceramic Brackets: An in-Vitro Comparative Study. J Clin Diagn Res. 2013;7(7):1495-7

5. Prabhakar R, Abinaya, Karthikeyan, Sarvanan, Vikram R. Comparision of shear bond strength of stainless steel and ceramic brackets at 24 hours after etching enamel with different proportions of acidulated phosphate fluoride. J Clin Diagn Res. 2014;8(8):ZC19-21 
Egyptian

Orthodontic Journal

6. Bishara SE. Ceramic brackets and the need to develop national standards. Am J Orthod Dentofacial Orthop. 2000;117(5):595-7

7. Bishara SE, Trulove TS. Comparisons of different debonding techniques for ceramic brackets: an in vitro study. Part II. Findings and clinical implications. Am J Orthod Dentofacial Orthop. 1990;98(3):263-73

8. Bishara SE, Fehr DE, Jakobsen JR. A comparative study of the debonding strengths of different ceramic brackets, enamel conditioners, and adhesives. Am J Orthod Dentofacial Orthop. 1993;104(2):170-9

9. Bishara SE, Fonseca JM, Boyer DB. The use of debonding pliers in the removal of ceramic brackets: force levels and enamel cracks. Am J Orthod Dentofacial Orthop. 1995;108(3):242-8

10. Grubisa HS, Heo G, Raboud D, Glover KE, Major PW. An evaluation and comparison of orthodontic bracket bond strengths achieved with self-etching primer. Am J Orthod Dentofacial Orthop. 2004;126(2):213-9

11. Bishara SE, VonWald L, Laffoon JF, Warren JJ. Effect of a self-etch primer/adhesive on the shear bond strength of orthodontic brackets. Am J Orthod Dentofacial Orthop. 2001;119(6):621-4

12. Hu H, Li C, Li F, Chen J, Sun J, Zou S, et al. Enamel etching for bonding fixed orthodontic braces. Cochrane Database Syst Rev. 2013;11:CD005516

13. Vertise Flow Technical Bulletin. 2011. Available from: http://kerrdental.com/vertiseflow.

14. Reynolds I, Von Fraunhofer J. Direct bonding of orthodontic brackets--a comparative study of adhesives. $\mathrm{Br} \mathrm{J}$ Orthod. 1976;3(3):143-6

15. Powers JM, Messersmith ML. Enamel etching and bond strength. In: Brantley WA, Eliades T, editors. Orthodontic Materials: Scientific and Clinical Aspects. Germany: Stuttgart: Thieme; 2001. p. 105-22. 
16. Retief D. Failure at the dental adhesive-etched enamel interface. J Oral Rehabil. 1974;1(3):265-84

17. Mirzakouchaki B, Kimyai S, Hydari M, Shahrbaf S, MirzakouchakiBoroujeni P. Effect of self-etching primer/adhesive and conventional bonding on the shear bond strength in metallic and ceramic brackets. Med Oral Patol Oral Cir Bucal. 2012;17(1):e164-70

18. İSMAN E, KARAARSLAN EŞ, OKŞAYAN R, TUNÇDEMİR AR, ÜŞÜMEZ S, Adanir $\mathrm{N}$, et al. Inadequate shear bond strengths of self-etch, self-adhesive systems for secure orthodontic bonding. Dent Mater J. 2012;31(6):947-53

19. Goracci C, Margvelashvili M, Giovannetti A, Vichi A, Ferrari M. Shear bond strength of orthodontic brackets bonded with a new self-adhering flowable resin composite. Clin Oral Investig. 2013; 17(2):609-17

20. Bishara SE, Olsen ME, Von Wald c L. Evaluation of debonding characteristics of a new collapsible ceramic bracket. Am J Orthod Dentofacial Orthop. 1997;112(5):552-9

21. Bishara SE, VonWald L, Laffoon JF, Jakobsen JR. Effect of altering the type of enamel conditioner on the shear bond strength of a resinreinforced glass ionomer adhesive. Am J Orthod Dentofacial Orthop. 2000;118(3):288-94

22. Fitzpatrick DA, Way DC. The effects of wear, acid etching, and bond removal on human enamel. Am J Orthod. 1977;72(6):671-81

23. Bishara SE, Fehr DE. Ceramic brackets: something old, something new, a review. Semin Orthod. 1997;3(3):178-88

24. Bishara SE, Fonseca JM, Fehr DE, Boyer DB. Debonding forces applied to ceramic brackets simulating clinical conditions. Angle Orthod. 1994;64(4):277-82

25. Diaz C, Swartz M. Debonding a new ceramic bracket: a clinical study. J Clin Orthod. 2004;38(8):442-5. 